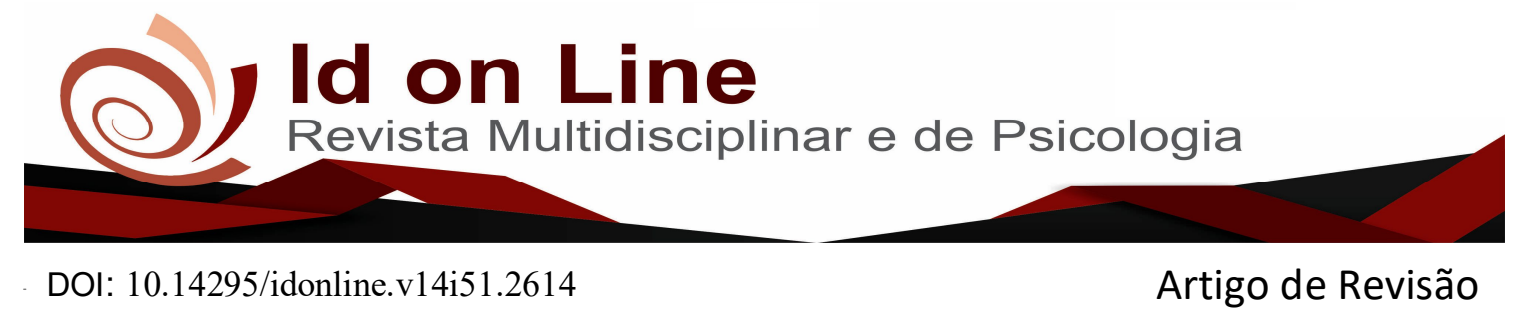

\title{
Distribuição da Mortalidade Materna no Estado da Paraíba no período de 2007 a 2016
}

\author{
Lívia Maria Costa Azevedo ${ }^{1}$, Damito Robson Xavier de Souza ${ }^{2}$, Kévia Katiúcia Santos Bezerra ${ }^{1,2}$, \\ Hermes Melo Teixeira Batista, ${ }^{3,{ }^{*},}$ Eduardo Sérgio Soares Sousa ${ }^{4}$, \\ Anne Milane Formiga Bezerra ${ }^{5}$, Eliane de Sousa Leite Ll.6 $^{1 .}$
}

Resumo: Objetivo: aliar a distribuição da mortalidade materna no estado da Paraíba no período de 2007 a 2016. Métodos: pesquisa do tipo documental e quantitativa, realizada com dados secundários obtidos a partir do banco de dados online e de acesso livre do Sistema de Informações sobre Mortalidade. A população foi composta por mulheres em idade fértil residentes no estado da Paraíba, que foram a óbito por morte materna no período de 2007 a 2016. Os dados foram analisados por estatística descritiva e confrontados com a literatura pertinente ao tema estudado. Resultados: ocorreram 324 óbitos, com maior predomínio durante a gravidez, o parto ou o aborto até 42 dias de puerpério $(74,3 \%)$ e realização de investigação (92\%), em mulheres na faixa etária entre 30 e 39 anos (41\%), de cor/raça parda $(74,4 \%)$, com escolaridade ignorada $(48,8 \%)$ e de estado civil solteira $(36,4 \%)$, em que o óbito resultou de causas diretas $(78,7 \%)$, destacando-se as síndromes hipertensivas específicas da gravidez (27,5\%), com predominância de mortes nas microrregiões de João Pessoa (24.4\%) e Campina Grande (14,8\%). Conclusão: o perfil de mortalidade materna vem merecendo atenção especial no Estado da Paraíba, devido á presença dos altos valores de razão da mortalidade materna, acompanhados de causas evitáveis na maioria dos casos.

Descritores: Mortalidade materna. Perfil epidemiológico. Sistema de informação em saúde.

\section{Distribution of Maternal Mortality in Paraiba State from 2007 to 2016}

\begin{abstract}
Objective: to evaluate the distribution of maternal mortality in the state of Paraíba from 2007 to 2016. Methods: a documental and quantitative research, with secondary data obtained from the online database and free access to the Mortality Information System . The population was composed of women of childbearing age living in the state of Paraíba, who died of maternal death in the period 2007 to 2016 . The data were analyzed by descriptive statistics and compared with the literature pertinent to the subject studied. Results: There were 324 deaths, with a higher prevalence during pregnancy, delivery or abortion up to 42 days postpartum (74.3\%) and research (92\%) among women aged between 30 and 39 years $(41$ (78.4\%), with schooling ignored (48.8\%) and single marital status $(36.4 \%)$, where death resulted from direct causes $(78.7 \%),(27.5 \%)$, with predominance of deaths in the microregions of João Pessoa (24.4\%) and Campina Grande (14.8\%). Conclusion: the maternal mortality profile has been receiving special attention in the state of Paraíba, due to the presence of high maternal mortality ratios, accompanied by preventable causes in the majority of cases.
\end{abstract}

Keywords: Maternal mortality. Epidemiological profile. Health information system.

\footnotetext{
${ }^{1}$ Universidade Federal de Campina Grande - UFCG, Cajazeiras, PB;

${ }^{2}$ Hospital Regional do Cariri, CE;

${ }^{3}$ Hospital Universitário Júlio Bandeira - UFCG/Ebserh;

${ }^{4}$ Doutorado em Sociologia pela UFPB, João Pessoa, PB. Brasil;

${ }^{5}$ Doutoradanda em Ciências da Saúde. Pela Faculdade de Ciências Médicas Santa Casa São Paulo, FCMSCSP, Brasil;

${ }^{6}$ Doutorado em Enfermagem pela Universidade Federal da Paraíba - UFPB;

${ }^{7}$ UNIFAP Araripina.

*Autor correspondente: hermes2710batista@gmail.com
} 


\section{Introdução}

A mortalidade materna é definida pela Classificação Internacional de Doenças e Problemas Relacionados à Saúde (CID-10) e pela Organização Mundial de Saúde (OMS) como a morte de uma mulher durante a gravidez ou em até 42 dias após o parto, independentemente da duração e do local da gestação, por qualquer causa relacionada ao período gestacional, sendo agravada por este ou pela sua gestão, excluindo-se os motivos acidentais ou incidentais. ${ }^{1,2}$

As mortes maternas podem ser classificadas em causa direta, as quais decorrem de complicações ocorridas durante a gravidez, o parto ou o puerpério por assistência inadequada à mulher; e em causa indireta, que se caracterizam pelo agravamento de doenças presentes na paciente, com início anterior a gravidez ou durante o seu curso, devido às alterações fisiológicas que esse período impõe no organismo materno. ${ }^{3}$

No Brasil, observa-se uma predominância em óbitos maternos de causa direta, notando os distúrbios hipertensivos da gestação, os sangramentos na gestação e as infecções como os seus principais fatores causadores, além de baixa renda e de baixa escolaridade, evidenciando, assim, as disparidades existentes na sociedade e se tornando uma preocupação na saúde pública. $3-5$

A mortalidade materna é considerada como o reflexo da inadequada assistência à saúde da mulher e do desrespeito aos seus direitos sexuais e reprodutivos, demonstrando que a sua ocorrência no país é um evento alarmante e que necessita da adoção de medidas públicas para sua diminuição, já que a morte materna pode ser evitada em $92 \%$ dos casos. Além disso, os países emergentes, como Serra Leoa, Nigéria e Somália, possuem as maiores taxas de mortalidade materna no mundo. ${ }^{6}$

Dada a importância e as dificuldades de redução nas taxas de mortalidade materna, principalmente nos países de renda média e baixa, a Organização das Nações Unidas (ONU), aprovou os "objetivos de desenvolvimento do milênio" (ODM) em 2000, na Declaração do Milênio, apresentando o quinto objetivo que versa sobre a "melhoria da saúde da mulher" e traz como um de seus componentes, a redução da razão da mortalidade materna (RMM) em três quartos até 2015. Os ODM foram estabelecidos com a finalidade de garantir o desenvolvimento sustentável da sociedade e a especial atenção aos graves problemas que afetam a humanidade. 1,7

A RMM é definida como o número de mortes maternas por 100.000 nascidos vivos (NV), sendo utilizada como indicador de saúde para avaliar a mortalidade materna, bem como 
para verificar as características de seus dados, relacionando-os com o período e com o local em que os óbitos aconteceram. ${ }^{8,9}$

A diminuição da RMM está intimamente relacionada com a acessibilidade precoce da mulher, seja na gravidez ou no puerpério, aos serviços de saúde que ofertem auxílio e suporte de qualidade, com atendimento completo e encaminhamento, quando necessário, a níveis complexos de atenção à saúde de acordo com o risco que seu quadro clínico impõe. ${ }^{1,10-11}$

No Brasil, os óbitos maternos caíram de 120 mortes por 100.000 NV em 1990 para 69 mortes por 100.000 NV em 2013, observando, assim, uma diminuição de 43\% na RMM. Entretanto, mesmo com essa redução, o país não atingiu a meta estabelecida pelo ODM, a qual seria de uma RMM igual ou inferior a 35 mortes por $100.000 \mathrm{NV}$ em $2015 .{ }^{10}$

A região Nordeste apresentou, no intervalo entre 2001 a 2012, uma diminuição de três óbitos maternos por $100.000 \mathrm{NV}$ a cada ano. Entretanto, suas taxas continuaram elevadas. No período de 2001 a 2007, apresentou entre 100 e 120 mortes por $100.000 \mathrm{NV}$. Este valor, agregado ao apresentado pela região Sul, que foi de 80 a 100 mortes por $100.000 \mathrm{NV}$, configuram-se como os maiores valores apresentados. Esta realidade nordestina se manteve no período de 2008 a 2012, cuja RMM se apresentou elevada com valores entre 60 e 80 mortes por $100.000 \mathrm{NV}$, sendo seguida pela região Centro-Oeste cujos valores são de 80 a 100 mortes por $100.000 \mathrm{NV} .^{1}$

Em posse do conhecimento que a região Nordeste apresenta altas taxas de mortalidade materna, justifica-se a relevância desta pesquisa pela necessidade de caracterizar o perfil de óbitos maternos no estado da Paraíba nos últimos 10 anos, de identificar as suas causas e de descrever as características relacionadas a condições sociodemográficas e gineco-obstétricas, a fim de possibilitar o desenvolvimento de ações e estratégias direcionadas para a prevenção de agravos e promoção da saúde nesse público.

Assim, o presente estudo tem por objetivo avaliar a distribuição da mortalidade materna no estado da Paraíba no período de 2007 a 2016.

\section{Métodos}

Trata-se de um estudo documental e quantitativo, realizado com mulheres em idade fértil residentes no Estado da Paraíba, que foram a óbito por morte materna no período entre 01 
de janeiro de 2007 a 31 de dezembro de 2016, compreendendo os indivíduos do sexo feminino com idade entre 10 e 49 anos.

Os dados sobre a mortalidade materna foram obtidos a partir do Sistema de Informações sobre Mortalidade do estado da Paraíba (SIM), disponível no endereço eletrônico http:/tabnet.datasus.gov.br/cgi/deftohtm.exe?sim/cnv/mat10PB.def, e do Sistema de Informações sobre Nascidos Vivos (SINASC), por meio do endereço eletrônico http://tabnet.datasus.gov.br/cgi/deftohtm.exe?sinasc/cnv/nvPB.def.

Para a coleta de dados foram utilizadas as seguintes variáveis no SIM: óbitos maternos, óbito na gravidez e no puerpério, óbito investigado, ano do óbito, faixa etária, cor/raça, escolaridade, estado civil, microrregião, categoria da décima revisão da Classificação Internacional das Doenças CID-10 e tipo de causa obstétrica; e do SINASC, utilizando as variáveis: nascidos vivos segundo o local de residência da mãe e ano do nascimento, para o cálculo da RMM.

Os dados foram analisados por estatística descritiva e distribuídos em gráficos e tabelas, através do programa Microsoft Office Excel for Windows 2010 e foram confrontados com a literatura pertinente ao tema estudado. Os dados utilizados para a realização do estudo são de domínio público, disponíveis de forma livre e gratuita na rede mundial de computadores por meio do DATASUS, sendo, assim, dispensado pela Comissão Nacional de Ética em Pesquisa em Seres Humanos. Contudo, todos os preceitos éticos estabelecidos pela Resolução ${ }^{\circ}$ 466/12 do Conselho Nacional de Saúde foram respeitados.

\section{Resultados}

A Tabela 1 apresenta o perfil sociodemográfico dos óbitos maternos na Paraíba no período de 2007 a 2016. Foi evidenciado um maior predomínio de mortes na faixa etária entre 30 e 39 anos, representando $41 \%$ do total de óbitos, na cor/raça parda com $74,4 \%$, na escolaridade ignorada com $48,8 \%$ e no estado civil solteira com $36,4 \%$. 
Tabela 1 - Perfil sociodemográfico dos óbitos maternos na Paraíba no período de 2007 a 2016.

\begin{tabular}{|c|c|c|}
\hline Variáveis & $\mathbf{N}$ & $\%$ \\
\hline \multicolumn{3}{|l|}{ Faixa etária } \\
\hline $10-14$ anos & 3 & 0,9 \\
\hline $15-19$ anos & 45 & 13,9 \\
\hline $20-29$ anos & 124 & 38,3 \\
\hline $30-39$ anos & 133 & 41,0 \\
\hline $40-49$ anos & 19 & 5,9 \\
\hline \multicolumn{3}{|l|}{ Cor/Raça } \\
\hline Parda & 241 & 74,4 \\
\hline Branca & 53 & 16,4 \\
\hline Preta & 17 & 5,2 \\
\hline Amarela & 1 & 0,3 \\
\hline Indígena & 1 & 0,3 \\
\hline Não informado & 11 & 3,4 \\
\hline \multicolumn{3}{|l|}{ Escolaridade } \\
\hline Nenhuma & 11 & 3,4 \\
\hline $1-3$ anos & 36 & 11,2 \\
\hline $4-7$ anos & 62 & 19,1 \\
\hline $8-11$ anos & 47 & 14,5 \\
\hline 12 anos e mais & 10 & 3,0 \\
\hline Ignorado & 158 & 48,8 \\
\hline \multicolumn{3}{|l|}{ Estado civil } \\
\hline Solteira & 118 & 36,4 \\
\hline Casada & 93 & 28,7 \\
\hline Separada judicialmente & 3 & 0,9 \\
\hline Viúva & 1 & 0,3 \\
\hline Outro & 36 & 11,2 \\
\hline Ignorado & 73 & 22,5 \\
\hline Total & 324 & 100,0 \\
\hline
\end{tabular}

O Gráfico 1 apresenta os dados relacionados à frequência de óbitos maternos no período de 2007 a 2016 no estado da Paraíba, correspondendo a 324 casos nos últimos 10 anos. Foram observados valores menores no período de 2007 a 2012, com um pico em 2009 de 33 mortes, e valores maiores no período de 2014 a 2016, com dois picos, um em 2013 de 40 mortes e outro em 2016 de 49 mortes. 
Gráfico 1 - Frequência de óbitos maternos na Paraíba no período de 2007 a 2016.


Fonte: DATASUS, 2017.

O Gráfico 2 apresenta os valores de RMM no período de 2007 a 2016 no estado da Paraíba. A maior RMM foi demonstrada no ano de 2016 (87,3 mortes por 100.000 NV), e a menor no ano de 2007 (33,6 mortes por 100.000 NV), analisando todo o período estudado. Foi observada uma tendência de aumento da RMM, com incremento de 53,7 óbitos maternos por 100.000 NV no ano de 2016, quando comparado com o ano de 2007, correspondendo a um aumento de $61,5 \%$, e, consequentemente não alcançando a redução de $75 \%$ estabelecida como meta pelo quinto ODM.

Gráfico 2 - Razão de mortalidade materna na Paraíba no período de 2007 a 2016.

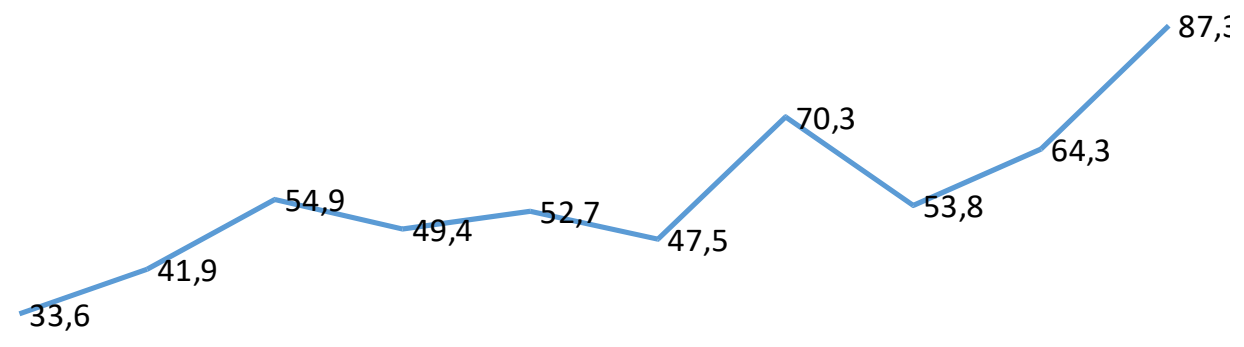

$\begin{array}{llllllllll}2007 & 2008 & 2009 & 2010 & 2011 & 2012 & 2013 & 2014 & 2015 & 2016\end{array}$

Fonte: DATASUS, 2017. 
Na Tabela 2 estão expostas as informações sobre as características dos óbitos maternos. Observou-se um maior número de óbitos por causas maternas com a porcentagem de 74,3\%, que demonstrou a morte materna durante a gravidez, o parto ou o aborto e até 42 dias de puerpério. Além disso, é importante destacar que ainda é grande o número de casos não informados ou ignorados, o que evidenciou a fragilidade na coleta de dados. Em relação à investigação dos óbitos, observa-se que a maioria foi investigada com o total de $92 \%$, sendo $77,2 \%$ com ficha síntese informada.

Tabela 2 - Características dos óbitos maternos na Paraíba no período de 2007 a 2016.

\begin{tabular}{lcc}
\hline Variáveis & $\mathbf{N}$ & $\mathbf{\%}$ \\
\hline Óbito na gravidez e no puerpério & & \\
Durante a gravidez, parto ou aborto & 95 & 29,3 \\
Durante o puerpério até 42 dias & 146 & 45,0 \\
Durante o puerpério de 43 dias a 1 ano & 6 & 1,9 \\
Não na gravidez ou no puerpério & 12 & 3,7 \\
Período informado inconsistente & 32 & 9,9 \\
Não informado ou ignorado & 33 & 10,2 \\
Óbito investigado & & \\
Sim, com ficha síntese informada & 250 & 77,2 \\
Sim, sem ficha síntese informada & 48 & 14,8 \\
Não investigado & 26 & 8,0 \\
Total & $\mathbf{3 2 4}$ & $\mathbf{1 0 0 , 0}$ \\
\hline
\end{tabular}

Fonte: DATASUS, 2017.

A Tabela 3 apresenta os dados relacionados aos tipos de mortes maternas por causas obstétricas, com um maior predomínio de morte materna por causa obstétrica direta, a qual decorre de complicações ocorridas durante a gravidez, o parto ou o puerpério por assistência inadequada à saúde da mulher, representando $78,7 \%$ do total de óbitos.

\begin{tabular}{lcc} 
Tabela 3 - Tipos de mortes maternas por causas obstétricas na Paraíba no período de 2007 a 2016. \\
\hline Variáveis & $\mathbf{N}$ & $\mathbf{\%}$ \\
\hline Tipos de causas obstétricas & & \\
Morte materna obstétrica direta & 255 & 78,7 \\
Morte materna obstétrica indireta & 55 & 17,0 \\
Morte materna obstétrica não especificada & 14 & 4,3 \\
Total & $\mathbf{3 2 4}$ & $\mathbf{1 0 0 , 0}$ \\
\hline
\end{tabular}

Fonte: DATASUS, 2017

Em relação à distribuição das causas de mortalidade materna na Paraíba no período de 2007 a 2016, trabalhou-se com elas divididas em grupos de doenças, utilizando as categorias do CID-10. Observou-se maior prevalência de óbitos por síndromes hipertensivas específicas

492 Id on Line Rev. Mult. Psic. V.14, N. 51 p. 486-501, Julho/2020 - ISSN 1981-1179 Edição eletrônica em http://idonline.e mnuvens.com.br/id 
da gravidez (27,5\%), as quais representam a hipertensão pré-existente à gravidez, a préeclâmpsia, a eclampsia e a hipertensão não especificada; por infecções na gravidez e no puerpério $(17,3 \%)$, as quais incluem a infecção pelo HIV, a infecção do trato geniturinário na gravidez, as infecções puerperais e as doenças infecciosas e parasitárias maternas; e por outras doenças da mãe que complicam a gravidez, o parto e o puerpério (13,0\%), conforme representado na Tabela 4 .

Tabela 4 - Causas de mortalidade materna na Paraíba no período de 2007 a 2016.

\begin{tabular}{lcc}
\hline Causa & $\mathbf{N}$ & $\mathbf{\%}$ \\
\hline Síndromes hipertensivas específicas da gravidez & 89 & 27,5 \\
Infecções na gravidez e no puerpério & 56 & 17,3 \\
Outras doenças da mãe que complicam a gravidez o parto e o puerpério & 42 & 13,0 \\
Complicações do trabalho de parto e do parto & 33 & 10,2 \\
Sangramento na gravidez, parto e pós parto & 30 & 9,3 \\
Gravidez que termina em aborto & 15 & 4,6 \\
Morte obstétrica por causa não especificada & 14 & 4,3 \\
Complicações do puerpério não classificadas em outras partes & 13 & 4,0 \\
Outros & 10 & 3,0 \\
Total & $\mathbf{3 2 4}$ & $\mathbf{1 0 0 , 0}$ \\
\hline
\end{tabular}

Fonte: DATASUS, 2017.

Mediante a análise da Tabela 5, percebe-se que as microrregiões paraibanas com os maiores índices de mortalidade materna entre os anos de 2007 e 2016 foram João Pessoa $(24,4 \%)$ e Campina Grande (14,8\%).

Tabela 5 - Óbitos maternos nas microrregiões da Paraíba no período de 2007 e 2016.

\begin{tabular}{lcc}
\hline Microrregião & $\mathbf{N}$ & $\mathbf{\%}$ \\
\hline João Pessoa & 79 & 24,4 \\
Campina Grande & 48 & 14,8 \\
Brejo & 15 & 4,6 \\
Cajazeiras & 13 & 4,0 \\
Curimataú Ocidental & 13 & 4,0 \\
Curimataú Oriental & 12 & 3,7 \\
Cariri Ocidental & 12 & 3,7 \\
Serra Teixeira & 12 & 3,7 \\
Sousa & 12 & 3,7 \\
Seridó Oriental & 11 & 3,4 \\
Itabaiana & 11 & 3,4 \\
Sapé & 11 & 3,4 \\
Catolé do Rocha & 10 & 3,0 \\
Patos & 10 & 3,0 \\
Guarabira & 10 & 3,0 \\
Litoral Norte & 8 & 2,5
\end{tabular}




\begin{tabular}{lcc} 
Litoral Sul & 8 & 2,5 \\
Esperança & 7 & 2,2 \\
Piancó & 6 & 1,9 \\
Itaporanga & 5 & 1,6 \\
Seridó Ocidental & 4 & 1,3 \\
Cariri Oriental & 4 & 1,3 \\
Umbuzeiro & 3 & 0,9 \\
Total & $\mathbf{3 2 4}$ & $\mathbf{1 0 0 , 0}$ \\
\hline
\end{tabular}

Fonte: DATASUS, 2017.

\section{Discussão}

O perfil sociodemográfico dos óbitos maternos na Paraíba no período de 2007 a 2016 apresentou um maior predomínio de mortes na faixa etária entre 30 e 39 anos, na cor/raça parda, na escolaridade ignorada e no estado civil solteira, demonstrando o menor acesso à assistência desses grupos populacionais, e, consequentemente, as injustiças sociais que estes enfrentam. 12,13

Sobre a faixa etária, dados semelhantes foram evidenciados por estudo ${ }^{12}$ realizado na Região de Saúde em Jequié - BA, o qual identificou a faixa etária entre 30 e 39 anos com o maior número de mortes, demonstrando que há predominância em mulheres mais maduras e mais predispostas à gravidez de risco, grupo que apresenta a necessidade de maior atenção à saúde.

Foi observado que a raça parda representa a maioria do número total de óbitos maternos, refletindo as dificuldades que as mulheres pardas apresentam ao acesso à assistência à saúde de qualidade, fato que denuncia a desigualdade racial existente no país. ${ }^{14}$

Nesse presente estudo, foi encontrado um predomínio de resultado ignorado na variável escolaridade, demonstrando inadequado preenchimento da DO, e sendo motivo de preocupação na verdadeira identificação do grupo mais susceptível à mortalidade materna, pois o correto preenchimento dessa variável fornece informações importantes que influenciam na análise da mortalidade materna. ${ }^{14}$

A escolaridade está relacionada com diversos fatores, tais como: idade materna na gravidez, adesão à assistência pré-natal e via de parto, e isso é comprovado, pois estudo demonstra que: há um menor número de anos de estudo entre as mães mais jovens, há um diminuído interesse de acesso aos serviços de saúde nas mulheres com baixa escolaridade e menos partos cesáreos entre essas mulheres. ${ }^{15-17}$ 
O número elevado de mortes maternas entre as mães solteiras apresenta a necessidade de uma atenção individualizada a esse grupo de mulheres, já que a falta de apoio familiar, demonstrada pela falta de um companheiro na realização de uma paternidade responsável, está associada a consequências adversas na morbidade e mortalidade materna. ${ }^{17}$

Em relação à avaliação da taxa de mortalidade materna no estado da Paraíba no período de 2007 a 2016, foi evidenciado que ao longo dos anos houve um aumento no número de óbitos, sendo registradas 20 mortes em 2007, enquanto em 2016, foram 49 óbitos, o que corresponde a um aumento de $40,8 \%$ em apenas 10 anos.

Embora tenham ocorridos inúmeros avanços nas condições de vida da população brasileira durante esse período, alguns aspectos ainda perduram como entraves, sobretudo do ponto de vista da saúde da mulher. A distribuição espacial dos territórios com altos índices de pobreza permanece concentrada nas Regiões Norte e Nordeste, o que intensifica as desigualdades sociais e reflete diretamente sobre a oferta de uma assistência segura e de qualidade, principalmente em municípios menores, o que requer o deslocamento dos indivíduos para outras localidades e estados. ${ }^{18}$

Neste sentido, a elevação do número de mortes maternas poderia estar relacionada às desigualdades regionais de saúde existentes no estado da Paraíba, as quais geram influência sobre o aumento das complicações na gravidez, parto ou puerpério. ${ }^{19}$ No panorama ampliado do Brasil, a taxa de mortalidade materna se caracteriza por uma inclinação na queda no número total de óbitos, demonstrada através da diminuição de 543 mil óbitos em 1990 para 287 mil óbitos no ano de 2010, reduzindo, assim, 47\% do total de óbitos. Entretanto, o país não alcançou a meta do quinto ODM, já que este preconizou uma redução de 5,5\% ao ano da taxa de mortalidade materna e só alcançou uma diminuição anual de apenas 3,1\%. ${ }^{18,19}$

A Região Nordeste se caracterizou com taxas elevadas de RMM nos anos de 2000 a 2009, com o valor médio de 64,01 mortes maternas por $100.000 \mathrm{NV}$, apresentando-se maior que a nacional, a qual apresentou uma RMM de 54,83 por $100.000 \mathrm{NV}$ no mesmo período. Além disso, foi demonstrado que, em 2009, o Nordeste apresentou um valor de RMM superior às demais Regiões do Brasil: Norte, 67,26; Centro-Oeste, 62,22; Sudeste, 62,09; e Sul 55,41. ${ }^{20}$ Foram evidenciados altos valores da RMM no estado da Paraíba no período de 2007 a 2016, o que apresenta concordância com os elevados números encontrados na Região Nordeste em estudo.

Esse achado poderia ser decorrente do reduzido percentual de gestantes que realizaram seis ou mais consultas de pré-natal, dentre as quais se destacam as mulheres com baixo nível 
socioeconômico e menos escolarizadas, ${ }^{20}$ corroborando com a população encontrada no presente estudo. Além disso, outros aspectos podem ter influenciado para essa elevação da RMM no período investigado, como o início tardio do acompanhamento pré-natal, a falta de solicitação ou de vagas para a realização de exames laboratoriais e clínico-obstétricos padronizados e a falta de orientações de educação em saúde acerca dos cuidados necessários durante a gestação. ${ }^{18-20}$

Observou-se uma predominância de mortes maternas na gravidez, no parto e no puerpério até 42 dias, sendo caracterizada pelas complicações no intercurso do ciclo gravídicopuerperal, o que demonstra a necessidade de uma assistência à saúde de qualidade nesse período como importante fator de prevenção da mortalidade materna, através do reconhecimento precoce dessas complicações e da adoção de medidas intervencionistas no momento adequado. 21

No tocante a investigação dos óbitos, nota-se que $92 \%$ dos óbitos foram investigados, o que demonstra uma melhoria na sua investigação com o compromisso efetivo da vigilância e monitorização da morte materna pelos Comitês de Mortalidade Materna, que tem a finalidade de identificar as suas causas e promover medidas de saúde para diminuir a mortalidade materna no país (BRASIL, 2009). Essa investigação possibilita a classificação da causa básica da morte materna e a realocação de mortes classificadas anteriormente à investigação como maternas para suas verdadeiras causas básicas. ${ }^{12}$

Em relação às causas obstétricas de mortalidade materna no estado da Paraíba entre 2007 e 2016, há uma maior prevalência de causas diretas. Esse achado corrobora com a média de mortalidade por causas diretas no Brasil, a qual é representada por $66,7 \%$ de mortes maternas no país, demonstrando que a assistência à saúde das mães no período da gravidez, do parto e do puerpério é inadequada, observada através da má condução terapêutica diante de complicações no ciclo gravídico-puerperal. ${ }^{22}$

No tocante ao estado da Paraíba no período de 2007 a 2016, as principais causas de mortalidade materna foram relacionadas a síndromes hipertensivas específicas da gestação, infecções na gravidez e no puerpério, e outras doenças da mãe que complicam a gravidez, o parto e o puerpério.

Dados semelhantes foram observados no estudo de Vega (2018) realizado em São Paulo - SP, o qual identificou que a hipertensão arterial apresentou $22,5 \%$ dos óbitos maternos entre 1993 e 2016, compreendendo as mortes maternas causadas pela pré-eclâmpsia, eclâmpsia e hipertensão arterial sistêmica, atribuindo essas mortes a uma falta de identificação precoce de 
fatores de risco para o estabelecimento dessas comorbidades; bem como a inadequada adoção de medidas de prevenção, como o uso de ácido aceltisalicílico e a reposição de cálcio, o insuficiente rastreio de hipertensão prévia a gestação, e consequentemente, a falta de controle pressórico antes da ocorrência da gravidez. ${ }^{23}$

Além disso, conforme estabelece Vega (2018), as complicações do descontrole da pressão arterial são determinantes no número de mortes maternas, como a presença de síndrome HELLP (hemolysis, elevated liver enzymes and low platelet count). Esta situação merece atenção cautelosa, já que após a sua instalação, há um aumento significativo da morbidade e da mortalidade materna; como, também, a presença de eclâmpsia, já que 50\% das mortes maternas por acidente vascular encefálico acontecem após a sua instalação.

A segunda maior causa de mortes em mães paraibanas foram as infecções na gravidez e no puerpério, o que, conforme explicitam Lima et al. (2016), são consideradas como causas evitáveis de morte materna, perdendo em frequência apenas para as síndromes hipertensivas em estudos de 2008 e 2011.

Essas duas comorbidades estão relacionadas com um pior desfecho na saúde infantil, já que estão associadas com prematuridade e baixo peso ao nascer, os quais são fatores de risco para a mortalidade infantil. Precisa-se, assim, de um cuidado especial para melhorar o quadro de mortalidade materno-infantil no país, a fim de diminuir o número de mortes maternas evitáveis no país, e melhorar o prognóstico da saúde infantil, através da identificação e tratamento precoces dessas infecções. ${ }^{24}$

Sobre a variável de outras doenças da mãe que complicam a gravidez, o parto e o puerpério, esta foi considera a terceira maior causa de mortalidade materna no estado da Paraíba no período de 2007 a 2016, contrapondo o que foi exposto no estudo de Ferraz e Bordignon (2012), o qual demonstrou que essa era principal causa de mortalidade materna no Brasil entre 2000 e 2009, representando $17,10 \%$ do total de óbitos maternos.

Em relação à distribuição da taxa de mortalidade no Estado no período de 2007 a 2016 segundo a microrregião, foi observada maior prevalência de óbitos nas microrregiões de João Pessoa e Campina Grande, respectivamente. Este achado pode ser explicado por estas serem as regiões com maior nível de complexidade de atenção à saúde na Paraíba, recebendo, possivelmente, os casos de maior morbidade à saúde materna; e por serem as mais populosas dentre as demais microrregiões paraibanas, as quais juntas concentram $29,4 \%$ da população (IBGE, 2010), sendo desta forma, responsáveis pelos maiores números de mortes. 
Mediante o exposto, faz-se necessário que os profissionais da área de saúde sejam capacitados para identificar as comorbidades e possíveis complicações que acometem as gestantes, e atuar adequadamente diante de tais situações, tomando condutas adequadas precocemente, já que a diminuição da mortalidade materna está intimamente associada com o acesso das grávidas e das puérperas a uma assistência de qualidade em tempo favorável nos diversos níveis de complexidade existentes no país. ${ }^{24}$

As limitações do estudo estão relacionadas à utilização de dados secundários, uma vez que a subnotificação dos dados pode dificultar o real conhecimento acerca da distribuição da mortalidade materna no estado da Paraíba.

\section{Conclusões}

Nos últimos anos o perfil de mortalidade materna vem merecendo atenção especial no Brasil e no mundo, devido à presença dos altos valores de RMM, acompanhados de causas evitáveis na maioria dos casos. O Estado da Paraíba vem acompanhando esse processo, mas apresenta obstáculos, que estão presentes em todo o Brasil, como a exclusão social e econômica, o que dificulta o acesso à saúde e deixa a população menos favorecida de forma vulnerável e susceptível a agravos e doenças.

A importância deste estudo resulta da necessidade da efetivação de políticas voltadas para os principais motivos que levam aos óbitos maternos e que são passiveis de prevenção por meio da adoção de medidas, pelos profissionais de saúde, como a identificação precoce de riscos à saúde materna no atendimento e a adoção de condutas adequadas, intervindo assim, de maneira mais eficiente.

Além disso, o presente estudo ressalta a importância da notificação adequada das mortes maternas para a elaboração e adoção dessas políticas de saúde, o que pode ser realizado com a busca ativa das mortes ocorridas pelos gestores dos estabelecimentos de saúde, com a disponibilização de coleta de informação nos prontuários por estes para equipes de vigilância de óbitos, e com a conscientização dos profissionais que preenchem a DO, motivando-os a não assiná-la em branco ou deixá-la previamente assinada, e a verificar todos os seus campos se estão devidamente e corretamente preenchidos.

Ao final dessa pesquisa, constatou-se que os seus objetivos foram alcançados, com a identificação do perfil da mortalidade materna no estado da Paraíba no período de 2007 a 2016 e espera-se que os achados abordados possam colaborar com a adoção de estratégias de 
prevenção e promoção da saúde das mulheres, através do reconhecimento precoce de fatores de risco e complicações que afetam a saúde da mulher no ciclo gravídico-puerperal, e da realização precoce de condutas adequadas para uma assistência à saúde de qualidade às mães. Além disso, almeja-se auxiliar os profissionais e acadêmicos de saúde a terem uma visão mais abrangente da problemática.

\section{Referências}

1. Silva, B. et al. Mortalidade materna no Brasil no período de 2001 a 2012: tendência temporal e diferenças regionais. Rev Bras Epidemiol, v. 19, n. 3, p. 484-493, 2016. Disponível em <http://www.scielo.br/pdf/rbepid/v19n3/1980-5497-rbepid-19-0300484.pdf>. Acesso em: 03 abr. 2017. http://dx.doi.org/10.1590/1980-5497201600030002

2. Carroll, AE. Why Is US Maternal Mortality Rising? JAMA. 2017;318(4):321. doi:10.1001/jama.2017.8390

3. Lopes, F. et al. Mortalidade materna por síndromes hipertensivas e hemorrágicas em uma Maternidade-escola referência de Alagoas. Ciências Biológicas e de Saúde Unit. Alagoas, v. $4, \quad$ n.2, p. 149-162, 2017. Disponível em $<$ https://periodicos.set.edu.br/index.php/fitsbiosaude/article/view/4493/2611>. Acesso em: 18 ago. 2018. http://dx.doi.org/10.5712/rbmfc9(30)687

4. Petersen, E. E., Davis, N. L., Goodman, D., Cox, S., Mayes, N., Johnston, E., ... Barfield, W. (2019). Vital Signs: Pregnancy-Related Deaths, United States, 2011-2015, and Strategies for Prevention, 13 States, 2013-2017. MMWR. Morbidity and mortality weekly report, 68(18), 423-429. doi:10.15585/mmwr.mm6818e1

5. Hitimana, R., Lindholm, L., Mogren, I., Krantz, G., Nzayirambaho, M., Sengoma, J. S., \& Pulkki-Brännström, A. M. (2019). Incremental cost and health gains of the 2016 WHO antenatal care recommendations for Rwanda: results from expert elicitation. Health research policy and systems, 17(1), 36. doi:10.1186/s12961-019-0439-9

6. Carreno, I; Bonilha, A; Costa, J. Evolução temporal e distribuição espacial da morte materna. Rev Saúde Pública. v. 48, n.4, p. 662-670, 2014. Disponível em $<$ http://www.scielo.br/pdf/rsp/v48n4/pt_0034-8910-rsp-48-4-0662.pdf $>$. Acesso em: 03 abr. 2017. http://dx.doi.org/10.1590/S0034-8910.2014048005220

7. Adu-Bonsaffoh K., Gyamfi-Bannerman C., Oppong S.A., Seffah J.D. Determinants and outcomes of preterm births at a tertiary hospital in Ghana. Placenta, Volume 79, April 2019, Pages 62-67. https://doi.org/10.1016/j.placenta.2019.01.007

8. Anafcheh T, Yaghoubi Doust M, Mojadam M, Mirkazemi R, Khafaie MA. Temporal and spatial distribution of under-five mortality and factors associated with multiple cases of under-five deaths within a family in the rural area of Khuzestan, Southern Iran. Sci Rep. 2018 Dec 18;8(1):17930. doi: 10.1038/s41598-018-36438-5. 
9. Szwarcwald, C. et al. Estimação da razão de mortalidade materna no Brasil, 2008-2011. Cad. Saúde Pública. Rio de Janeiro, v. 30, p. 71-83, 2014. Disponível em $<$ http://www.scielo.br/pdf/csp/v30s1/0102-311X-csp-30-s1-0071.pdf $>$. Acesso em: 20 de jul. 2018. http://dx.doi.org/10.1590/0102-311X00125313

10. Vega, C.E.P. et al, Mortalidade materna tardia: comparação de dois comitês de mortalidade materna no Brasil. Cad. Saúde Pública 33 (3) 20 Abr 2017. https://doi.org/10.1590/0102311 X00197315

11. Forde I, Tripathi V. Association of Place of Residence and Under-Five Mortality in Middle- and Low-Income Countries: A Meta-Analysis. Children (Basel). 2018;5(4):51. Published 2018 Apr 18. doi:10.3390/children5040051

12. Mascarenhas, P. et al. Análise da mortalidade materna. Rev Enferm UFPE. v. 11, n. 11, p. 4653-4662, 2017. Disponível em < file://C:/Users/Guest/Downloads/231206-75297-1PB.pdf>. Acesso em 20 set. 2018. DOI: 10.5205/reuol.11138-99362-1SM.1111sup201715

13. Santana DS, Silveira C, Costa ML, et al. Perinatal outcomes in twin pregnancies complicated by maternal morbidity: evidence from the WHO Multicountry Survey on Maternal and Newborn Health. BMC Pregnancy Childbirth. 2018;18(1):449. Published 2018 Nov 20. doi:10.1186/s12884-018-2082-9

14. Souza, Maria de Lourdes de, Laurenti, Ruy, Knobel, Roxana, Monticelli, Marisa, Brüggemann, Odaléa Maria, \& Drake, Emily. (2013). Maternal mortality due to hemorrhage in Brazil. Revista Latino-Americana de Enfermagem, 21(3), 711-718. https://dx.doi.org/10.1590/S0104-11692013000300009

15. Laopaiboon $\mathrm{M}$ et al, Advanced maternal age and pregnancy outcomes: a multicountry assessment.WHO Multicountry Survey on Maternal Newborn Health Research Network. BJOG. 2014 Mar;121 Suppl 1:49-56. doi: 10.1111/1471-0528.12659.

16. Ministério da Saúde (BR). Secretaria de Vigilância em Saúde. Departamento de Análise de Situação de Saúde. Guia de Vigilância Epidemiológica do Óbito Materno. Brasília: Ministério da Saúde; 2018. Disponível em <http:// http://svs.aids.gov.br/dantps/centraisde-conteudos/publicacoes/saude-brasil/saude-brasil-2018-analise-situacao-saude-

segundo-perfil-mortalidade-estados-brasileiros-distrito-federal.pdf $>$. Acesso em: 05 jun. 2018 .

17. Ministério da Saúde, (BR). Secretaria de Vigilância em Saúde. Departamento de Análise de Situação em Saúde. Saúde Brasil 2018: uma análise da situação de saúde e das doenças transmissíveis relacionadas à pobreza. Brasília: Ministério da Saúde; 2014. Disponível em <http:/ http://svs.aids.gov.br/dantps/centrais-de-conteudos/publicacoes/saudebrasil/saude-brasil-2018-analise-situacao-saude-segundo-perfil-mortalidade-estadosbrasileiros-distrito-federal.pdf $>$. Acesso em: 22 set. 2018

18. Martins ACS, Silva LS. Epidemiological profi le of maternal mortality. 2018; 71(suppl 1):725-31. http://dx.doi.org/10.1590/0034-7167-2017-0624

19. Albuquerque MV, Viana ALA, Lima LD, Ferreira MP, Fusaro ER, Iozzi FL. Regional health inequalities: changes observed in Brazil from 2000-2016. Ciênc Saúde Colet. 2017; 
22(4):1055-64. Available from: http://www.scielo.br/pdf/csc/v22n4/en_1413-8123-csc22-04-1055.pdf

20. Tomasi E, Fernandes PAA, Fischer T, Siqueira FCV, Silveira DS, Thumé E et al. Qualidade da atenção pré-natal na rede básica de saúde do Brasil: indicadores e desigualdades sociais. Cad Saúde Pública. 2017; 33(3):e00195815. Disponível em: http://www.scielo.br/pdf/csp/v33n3/1678-4464-csp-33-03-e00195815.pdf

21. Melo, E; knupp, V. Mortalidade materna no município do Rio de Janeiro: magnitude e distribuição. Esc Anna Nery Rev Enferm. v. 12, n. 4, p. 773-779, 2008. Disponível em http://www.scielo.br/scielo.php?pid=S1414-

$81452008000400023 \&$ script $=$ sci_abstract\&tlng=pt. Acesso em 20 set. 2018. http://dx.doi.org/10.1590/S1414-81452008000400023.

22. Biano, R. et al. Mortalidade materna no Brasil e nos municípios de Belo Horizonte e Uberaba, 1996 a 2012. Revista de Enfermagem do Centro-Oeste Mineiro. v. 7, p. 1-10, 2017. Disponível em http://www.seer.ufsj.edu.br/index.php/recom/article/view/1464/1575>. Acesso em: 20 set. 2018. http://dx.doi.org/10.19175/recom.v7i0.1464

23. Dias, J.M.G. et al. Mortalidade Materna. Rev Med Minas Gerais 2015; 25(2): 173-179 DOI: $10.5935 / 2238-3182.20150034$.

24. Botelho, N. et al. Causas de morte materna no Estado do Pará, Brasil. Rev Bras Ginecol Obstet. v. 36, n. 7, Rio de Janeiro, 2014. Disponível em $<$ http://www.scielo.br/scielo.php?script=sci arttext\&pid=S0100-72032014000700290 $>$. Acesso em: 22 set. 2018. DOI: 10.1590/SO100-720320140004892

25. Moura, B. et al. Internações por complicações obstétricas na gestação e desfechos maternos e perinatais, em uma coorte de gestantes no Sistema Único de Saúde no Município de São Paulo, Brasil. Cad. Saúde Pública. v. 34, n.1, p. 1-13, 2018. Disponível em $<$ https://www.scielosp.org/pdf/csp/2018.v34n1/e00188016/pt>. Acesso em 22 set. 2018. doi: 10.1590/0102-311X00188016

26. Vega, C. Desafios na redução da mortalidade materna no Município de São Paulo. Rev Med. v. 97, n. 2, p. 235-243, 2018. Disponível em < https://www.revistas.usp.br/revistadc/article/view/143244/140805>. Acesso em: 28 de set. 2018. doi: http://dx.doi.org/10.11606/issn.1679-9836.v97i2p235-243

\section{Como citar este artigo (Formato ABNT):}

AZEVEDO, Lívia Maria Costa; SOUZA, Damito Robson Xavier de; BEZERRA, Kévia Katiúcia Santos; BATISTA, Hermes Melo Teixeira; SOUSA, Eduardo Sérgio Soares; BEZERRA, Anne Milane Formiga; LEITE, Eliane de Sousa. Distribuição da Mortalidade Materna no Estado da Paraíba no período de 2007 a 2016. Id on Line Rev.Mult.Psic., Julho/2020, vol.14, n.51, p. 486-501. ISSN: 19811179.

Recebido: 06/07/2020;

Aceito: $10 / 07 / 2020$. 